\title{
Novas práticas de atenção ao parto e os desafios para a humanização da assistência nas regiões sul e sudeste do Brasil
}

\author{
New childbirth practices and the challenges for the humanization \\ of health care in southern and southeastern Brazil
}

Ricardo Motta Pereira ${ }^{1}$

Giovanna de Oliveira Fonseca ${ }^{2}$

Ana Célia Cirino Costa Pereira ${ }^{2}$

Gabrielly Antunes Gonçalves ${ }^{2}$

Roberta Amaral Mafra ${ }^{2}$
${ }^{1}$ Faculdade de Medicina de Ribeirão Preto,

Universidade de São Paulo. Av. Bandeirantes 3900

Monte Alegre. 14049-900

Ribeirão Preto SP Brasil.

rmpereira@alumni.usp.br

${ }^{2}$ Faculdade de Medicina,

Universidade José do

Rosário Vellano. Belo

Horizonte MG Brasil.
Abstract The humanization of care in childbirth and the choice of performing cesarean or vaginal delivery have long been discussed in Brazil and worldwide. The complexities of the factors surrounding this issue range from the quality of obstetric care through to the significance of childbirth for women. A new proposal for humanization of delivery was introduced by the Brazilian Ministry of Health, the objectives of which were to make changes to the current system of delivery practices regarding, access, care, quality and resolution, in order to make it a more human and less technical experience. The Sofia Feldman Hospital, in Belo Horizonte - MG, is a benchmark in the adoption of best practices in care during childbirth, according to the Brazilian National Health Agency. However, for the humanization to become a national reality, there are still many challenges to be overcome within the public health system and the private partnerships. The most important problems are related with the current education system that continues to prepare health professionals to act in an interventional way, focused on the physician figure. This study aims to provide an overview about the different humanized care practices focused on pregnancy and childbirth, conducted in southern and southeastern Brazil.

Key words Midwifery, Humanized childbirth, Perinatal care, Natural childbirth
Resumo Os diferentes modelos de assistência ao parto e a escolha pela realização de cesáreas ou parto vaginal há tempos são debatidos no Brasil e no mundo. A complexidade dos fatores que cercam o tipo de parto escolhido e sua assistência tem suscitado questionamentos que vão desde a qualidade da atenção obstétrica até o significado da parturição para as mulheres. Assim, a nova proposta do Ministério da Saúde (MS), de humanização na atenção ao parto estabelecendo mudanças em relação ao acesso, assistência, qualidade e resolutividade, tem por objetivo tornar a experiência da gestação mais humanizada e menos tecnicista. O Hospital Sofia Feldman, em Belo Horizonte (MG), é tido como referência pelas boas práticas nessa área, de acordo com a Agência Nacional de Saúde Suplementar. Para que a humanização do cuidado gestacional se torne uma realidade nacional ainda existem muitos desafios a serem superados dentro dos sistemas de saúde, como problemas, sobretudo quanto ao sistema de formação educacional, o qual continua a preparar profissionais de saúde dentro do modelo intervencionista, focado na figura do médico. O presente estudo tem por objetivo fornecer um panorama acerca das diferentes práticas assistenciais humanizadas, voltadas à gestação e ao parto, realizadas nas regiões sul e sudeste do Brasil.

Palavras-chave Assistência ao parto, Parto humanizado, Assistência pré-natal, Parto normal 


\section{Introdução}

Os diferentes modelos de assistência ao parto e a realização de cesárea ou parto vaginal, são questões debatidas há muito tempo e, particularmente no Brasil, desde a década de $80^{1,2}$. De acordo com Barbosa et al. ${ }^{3}$, a complexidade dos fatores que cercam o tipo de parto escolhido e sua assistência tem suscitado questionamentos envolvendo desde a qualidade da atenção obstétrica até o significado da parturição para as mulheres. Segundo a Organização Mundial de Saúde (OMS), o objetivo da assistência ao parto é garantir a saúde de mulheres e recém-nascidos, com o mínimo de intervenções médicas, buscando a segurança de ambos. Dessa maneira, a OMS recomenda que o profissional de saúde intervenha no nascimento de uma criança somente quando necessário. Apesar dessa recomendação, a incidência do parto cesáreo está aumentando em diversos países, inclusive no Brasil $1^{4}$.

A cesárea é uma intervenção cirúrgica originalmente concebida para reduzir o risco de complicações maternas e/ou fetais durante a gravidez e o trabalho de parto ${ }^{1}$. Essa intervenção possui riscos, a despeito das melhorias na segurança dessa cirurgia. Antes só realizada em mulheres mortas para salvar a vida do feto, a cesariana passou a proporcionar segurança à gestante e a seu filho em situações de maior complexidade ${ }^{2}$. Embora a maioria dos autores concorde que a cesárea deve ser evitada na ausência de indicação médica, estudos, como o realizado por Lurie ${ }^{5}$, relatam que melhorias nas técnicas cirúrgicas, medidas de prevenção de infecção e transfusões sanguíneas permitiriam indicar o procedimento também para a satisfação dos anseios da mãe e/ ou da família.

De acordo com Bergholt et al. ${ }^{4}$, no Brasil há um predomínio do modelo de atenção ao parto definido como evento médico ou tecnológico, segundo o qual a gestante é tratada como paciente onde o médico é o profissional responsável pela execução do parto em ambiente hospitalar e o parto cesáreo é predominantemente o mais realizado. Com o intuito de reverter essa situação, a Agência Nacional de Saúde Suplementar (ANS) está buscando experiências bem-sucedidas no estímulo ao parto normal e que possam servir de modelos a serem seguidos. Nesse sentido, a ideia da ANS é que os hospitais credenciados aos planos de saúde promovam uma mudança nos modelos de atenção ao parto, e que em longo prazo, o nascimento mais saudável diminua as taxas de cesarianas sem indicação clínica, as internações em UTIs neonatais e as taxas de prematuridade e aumente a satisfação das mulheres com a atenção recebida ${ }^{6}$.

Frente a essa realidade, subentende-se que a assistência obstétrica necessita de uma ampla mudança, a qual contemple seus aspectos de: acesso, acolhimento, qualidade e resolutividade. A proposta de humanização do parto vem reconhecer a autonomia da mulher enquanto ser humano, e da óbvia necessidade de tratar esse momento com práticas que, de fato, tenham evidências e permitam aumentar sua segurança e bem-estar, bem como do recém-nascido ${ }^{7}$. Diante deste pressuposto de necessidade de mudança nos paradigmas que permeiam a assistência ao parto, na capital mineira, o Hospital Sofia Feldman, é tido como referência pelas boas práticas na área de atenção ao parto e nascimento ${ }^{6}$. O hospital segue diretrizes do Ministério da Saúde (MS) como atenção ao parto realizada por equipes multidisciplinares com enfermeiras obstetras e médicos, estímulo à participação de acompanhante, oferta de medidas não farmacológicas para alívio da dor e atenção ao pré-parto, parto e pós-parto em um único ambiente.

O cenário de referência de qualidade em serviços prestados à saúde da gestante que caracteriza o hospital mineiro sugere a hipótese de que as mudanças nas práticas de atenção ao parto, preconizado pelo MS, ao tornar essa experiência mais humanizada e menos tecnicista traz inúmeros benefícios ${ }^{6}$. Neste sentido, as práticas realizadas no Hospital Sofia Feldman e seus benefícios justificariam a implantação nacional de um novo modelo humanizado de atenção ao parto. Entretanto, pouco se sabe sobre como esse processo de mudança na assistência tem ocorrido nas regiões sul e sudeste do Brasil, áreas de maior concentração populacional e que exercem grande influência sobre todo território nacional. Diante disso, o presente estudo tem por objetivo fornecer um panorama acerca das diferentes práticas assistenciais humanizadas, voltadas à gestação e ao parto, realizadas nas regiões sul e sudeste do Brasil.

\section{Material e métodos}

Tratou-se de uma revisão narrativa da literatura sobre os modelos assistenciais ao parto e às práticas humanizadas empregados nas regiões sul e sudeste do Brasil. Para tanto, buscou-se artigos nas principais bases de pesquisa cientifica como: Scielo, Google Acadêmico, Periódicos Capes, Bireme e PubMed; trabalhos relacionados ao 
A partir dos anos 2000 foi introduzida pelo MS, na política pública federal, a proposta de $\mathrm{Hu}$ manização na Atenção a Nascimentos e Partos com o objetivo de realizar mudanças no sistema atual de atenção ao parto 7 . De acordo com Rattner $^{7}$ o sentido do termo humanização adotado foi o de equidade / cidadania o qual garante a toda gestante o direito ao atendimento pré-natal integral e completo - mínimo de seis consultas e a de puerpério, todos os exames preconizados, vacinação antitetânica e garantia de vaga para o parto. O MS preconiza que haverá estímulos financeiros para que municípios qualifiquem profissionais e a efetividade da realização de pré-natais e que os hospitais garantam vaga para gestantes inscritas no Programa de Humanização do Pré-Natal e Nascimento - PHPN. Além disso, outras iniciativas do MS incluem: campanhas pelo parto normal humanizado, com presença de acompanhante, e pela redução das cesáreas desnecessárias ${ }^{7}$.

De acordo com Rattner ${ }^{7}$ essa busca por mudanças no atual modelo de atenção ao parto, bem como o surgimento do movimento pela humanização do parto e nascimento, teve seu início há 25 anos. No entanto, para que a humanização na atenção a nascimentos e partos se torne uma realidade cotidiana nos centros de serviços, ainda existem inúmeros e importantes desafios para a gestão do sistema. Os principais problemas sérios dizem respeito às mudanças preconizadas, sobretudo no sistema de saúde atual o qual ainda continua preparando profissionais dentro do modelo intervencionista, considerado inadequado por especialistas? ${ }^{7}$.

Segundo este mesmo autor, o grande desafio para a implantação de um modelo baseado em práticas humanizadas diz respeito à articulação entre os Ministérios da Saúde e da Educação, para que a grade curricular dos cursos de saúde incorpore além dos fundamentos da "Medicina Baseada em Evidências", uma visão humana imprescindível ao bom exercício profissional. De acordo com Dias e Domingues ${ }^{8}$, os quais defendem a proposta de fortalecer o modelo de atenção multiprofissional, o grande desafio para

todos os profissionais que prestam assistência a parturientes é o de minimizar o sofrimento, tornando a vivência do trabalho de parto uma experiência de crescimento e realização para a mulher e sua família.

Em estudo retrospectivo realizado por Borges et al. ${ }^{9}$, no Hospital Sofia Feldman, evidenciou os benefícios do uso de terapias humanizadas integrativas complementares no período gravídico e puerperal. Este estudo teve como objetivo caracterizar as usuárias do hospital e as terapias integrativas utilizadas e a sua percussão no alívio de dor e estresse. Foram realizadas entrevistas padronizadas com 105 mulheres usuárias do serviço às quais identificaram que as práticas integrativas mais utilizadas foram: musicoterapia, aromaterapia, oficinas de chá e o escalda pés. Os relatos das usuárias mostram grande aceitação das terapias alternativas e que estas proporcionam alívio e relaxamento diante do período de angustia que antecede e precede ao parto, além de fortalecer a mulher para o enfrentamento da situação vivenciada.

Esses resultados dão suporte à possibilidade de mudança no modelo assistencial do parto e puerperal, antes somente biomédico e intervencionista, agora para uma assistência holística que valoriza o autocuidado, que é barato e humanizado. Além disso, os resultados positivos do estudo indicam a necessidade de ampliação da disponibilidade de terapias integrativas complementares no âmbito intra-hospitalar, facilitando o acesso das parturientes a esses e outros recursos que promovam a experiência do parto como um ato mais fisiológico, humanizado e não puramente tecnicista.

Segundo a OMS, o parto de uma gestação de baixo risco pode ser assistido com segurança no domicílio, numa casa de parto ou na maternidade de um hospital, sendo a enfermeira-parteira o profissional mais adequado para essa função. Ainda de acordo com a OMS, parto de baixo risco tem início espontâneo entre 37 - 42 semanas completas, sem nenhum fator de risco identificado, mantendo-se esse quadro durante todo processo e que culmina com o nascimento de um recém-nascido em posição cefálica de vértice ${ }^{1,2}$.

Os chamados Centros de Parto Normal (CPN) surgiram com o objetivo de resgatar o direito à privacidade e à dignidade da mulher ao dar à luz num local semelhante ao seu ambiente familiar, oferecendo recursos tecnológicos apropriados em casos de eventual necessidade ${ }^{3}$.

Esses centros seguem um padrão de procedimentos previamente estabelecidos e que direcio- 
nam as ações que realizam de forma a atender as normas estabelecidas pelo MS. Constituem-se em unidades de atendimento ao parto normal, localizadas fora do centro cirúrgico obstétrico. Assim, os CPN dispõem de um conjunto de elementos destinados a receber a parturiente e seus acompanhantes, permitindo um trabalho de parto ativo e participativo, empregando práticas baseadas em evidências recomendadas e que os diferenciam dos serviços tradicionais de atenção obstétrica, de acordo com Machado e Praça ${ }^{10}$ Esses mesmos autores compactuam a ideia de que a assistência obstétrica deve envolver um conjunto de conhecimentos, de práticas e de atitudes que visam não só a promoção do parto, mas também um nascimento saudável e a prevenção da morbimortalidade materna. Apesar de, no Brasil, a assistência em CPN ser uma modalidade inovadora, estudos internacionais mostram resultados favoráveis à assistência gestacional em casas de parto ${ }^{11,12}$.

Na busca por evidências científicas acerca dos benefícios da assistência ao parto realizado fora do ambiente hospitalar, Campos e Lana ${ }^{13}$ realizaram um estudo analisando os principais achados clínicos da assistência prestada no CPN “Dr. David Capistrano da Costa Filho", o qual funciona integrado ao Hospital Sofia Feldman. A partir da entrevista com 2.117 parturientes de baixo risco, admitidas nesse CPN, foram analisadas as variáveis: transferência materna, tipo de parto, índice de APGAR no $1^{\circ}$ e $5^{\circ}$ minutos de vida, taxa de admissão em unidade neonatal, percentual de avaliações realizadas pelo obstetra ou pediatra, além das taxas de mortalidade neonatal. Ao final do estudo foi observada menor taxa de cesáreas, menor número de anormalidades cardíacas fetais, bem como uma maior satisfação dessas mulheres em relação à assistência tradicional.

Ainda em relação à assistência prestada nos CPN, um estudo do tipo metanálise realizado por Hodnett ${ }^{14}$ mostrou que mulheres atendidas nesses centros utilizaram em menor quantidade métodos farmacológicos para o alívio da dor, ocitocina, além de um menor número de episiotomias realizadas. Considerando o baixo número de estudos desta natureza realizados no Brasil, os indicadores assistenciais obtidos no CPN mineiro estão próximos dos resultados presentes na literatura internacional atual ${ }^{13}$. Contudo, mais estudos controlados e comparativos, entre a assistência hospitalar e a assistência nas $\mathrm{CPN}$, são necessários para a obtenção de resultados mais conclusivos.

Diante da necessidade de melhorar a capacidade de analisar a qualidade dos serviços volta- dos à atenção ao parto e a gestante, bem como o cuidado pré-natal, Puccini et al. ${ }^{15}$ realizaram um estudo em Embu das Artes - SP. O estudo foi realizado no ano de 1996, teve como objetivo avaliar a assistência ao pré-natal e ao parto de mães com filhos menores que um ano no presente município. Com uma amostra constituída por 483 crianças, segundo quatro estratos de condições de vida: tipo de moradia, o número de moradores, saneamento básico, a renda e a escolaridade dos chefes de família. Os resultados revelaram que a procura do serviço de saúde para o cuidado pré-natal e parto é praticamente universal na cidade, e a hipótese de que a população das áreas mais pobres, das favelas, estivesse sem assistência, não foi confirmada. Entretanto, foi observado que nessa parcela da população houve maiores dificuldades de acesso e deficiências quanto à qualidade da atenção, contribuindo, provavelmente, para uma maior frequência de intercorrências neonatais, talvez também de mortalidade, aspecto este não abordado neste estudo e constituindo um ponto fraco metodológico.

Com objetivo de analisar a qualidade da atenção pré-natal e ao parto, Neumann et al. ${ }^{16}$ realizaram um estudo transversal de base populacional em 2180 crianças menores de três anos residentes em Criciúma - SC. Os resultados mostraram que quase a totalidade das mães $(96,6 \%)$ realizaram pelo menos uma consulta de pré-natal e optaram pelo parto hospitalar. Por outro lado, mães de famílias com menor renda iniciaram o prénatal mais tarde e realizaram menos consultas. A população de baixa renda apresentou menor probabilidade de receber atendimento pré-natal adequado, seja por apresentar maior frequência de mães que não o fazem, seja por iniciar o pré-natal mais tardiamente, ou ainda por receber atendimento com menor qualidade técnica. Dados esses que corroboram aos encontrados na cidade de Embu das Artes - SP, fato esse que reforça a dificuldade de acesso entre os mais pobres. Nesse sentido, esforços devem ser feitos no sentido de captar precocemente as mães provenientes de famílias de menor poder aquisitivo e deve ser utilizado o enfoque de risco com discriminação positiva.

Com o intuito de descrever o perfil social e obstétrico das puérperas de uma maternidade no município da Serra - ES, Primo et al. ${ }^{17}$, realizaram um estudo com 1.335 mulheres da localidade. Os resultados mostram que escolaridade materna está fortemente associada ao número de consultas no pré-natal, além disso, verificouse que as mulheres com maior grau de instrução 
apresentaram um percentual maior de parto cesáreo. Com objetivo semelhante, Leite et al. ${ }^{18}$ procurou examinar a relação entre o número de consultas de pré-natal, tipo de parto e número de gestação e variáveis socioeconômicas em 323 mulheres de uma maternidade no município de São Mateus - ES. Os resultados mostraram significância estatística ao relacionar o número de consultas de pré-natal com a faixa etária, situação conjugal, raça/cor, renda familiar, escolaridade, ocupação e categoria de admissão. O tipo de parto apresentou significância estatística quando associado com as variáveis supracitadas. Diante do exposto, é possível inferir que as características socioeconômicas exercem influência no número de consultas de pré-natal e no tipo de parto.

A falta de atendimento ambulatorial no final da gestação, no momento de maior probabilidade de intercorrências obstétricas, é fator importante na determinação dos resultados maternos e perinatais ${ }^{1}$. Patah sugere que até algum tempo atrás era comum que o serviço ambulatorial de pré-natal orientasse a gestante, em sua fase final de gestação, a procurar um hospital no momento do parto deixando sob sua responsabilidade conseguir vaga para internação. Nesse panorama da situação obstétrica, a crença de que existe uma desumanização em um momento tão importante e, principalmente, o direito que toda mulher tem de garantia ao atendimento foram consideradas como questões emblemáticas a serem enfrentadas ${ }^{1}$.

D'Orsi et al. ${ }^{19}$ realizaram um estudo do tipo caso-controle com o objetivo de avaliar a qualidade da atenção durante o processo de trabalho de parto de acordo com as normas da OMS em maternidades do município do Rio de Janeiro de outubro de 1998 a março de 1999. A amostra foi composta por 461 mulheres oriundas de uma maternidade pública (230 partos vaginais e 231 cesáreas) e por 448 mulheres provenientes de uma maternidade conveniada (224 partos vaginais e 224 cesáreas). Os resultados mostraram uma baixa frequência de algumas práticas que devem ser encorajadas, principalmente no serviço particular como: presença de acompanhante, deambulação durante o trabalho de parto, aleitamento na sala de parto. Por outro lado, práticas comprovadamente danosas e que devem ser eliminadas também foram observadas no serviço privado como: uso de enema, tricotomia, uso rotineiro de ocitocina, restrição ao leito durante o parto e posição de litotomia para parto vaginal. A maternidade pública, apesar de atender clientela com maior risco gestacional, apresenta perfil menos intervencionista que maternidade conve- niada, entretanto, procedimentos realizados de maneira rotineira merecem ser discutidos à luz de evidências dos seus benefícios.

Ainda no município do Rio de Janeiro, um estudo realizado por Marque et al. ${ }^{20}$, com o objetivo de discutir a percepção de diferentes equipes de enfermagem sobre a humanização do parto e nascimento, realizaram um estudo por meio de entrevista com esses profissionais. As depoentes afirmaram que o parto feito sem manobras, adição de drogas como a ocitocina e o apoio da enfermagem na amamentação e a orientação no pré-natal são práticas de humanização ao parto e nascimento. Por outro lado, o uso de fórceps, ocitocina, a falta de cuidados e a negligência são maneiras de desumanizar a assistência. Esse estudo se torna particularmente importante uma vez que a percepção crítica do trabalho desempenhado por esses profissionais podem tornar mais humanizada essa assistência, pois suscita a necessidade de refletir sobre o tema, e isso pode conscientizá-los da importância de sua participação na assistência, educação, promoção da saúde, prevenção de intercorrências na gravidez e recuperação da saúde.

Considerando que a qualificação e a humanização são características essenciais da atenção obstétrica e neonatal a ser prestada pelos serviços de saúde e a necessidade de conhecer a assistência prestada às parturientes (569 partos, sendo 296 cesáreos e 273 vaginais), atendidas nos hospitais vinculados ao SUS no município de Maringá PR, um estudo de Nagahama e Santiago ${ }^{21}$ que teve por objetivo caracterizar essa assistência e identificar obstáculos e aspectos facilitadores para implantação do cuidado humanizado. Os dados apontaram para um modelo assistencial marcado pelo peso da herança higienista nas rotinas hospitalares e nas práticas profissionais, centrado no profissional médico como condutor do processo. Os fatores institucionais, identificados nas dificuldades de organização institucional e na estrutura física, as rotinas hospitalares e, sobretudo, a prática e postura individuais dos profissionais de saúde denotaram barreiras que, em seu conjunto, dificultam a implantação do modelo humanizado na assistência ao parto e nascimento.

Uma vez que o parto representa um marco na vida da mulher, repercutindo profundamente nos seus planos físico, emocional e social, um estudo realizado por Lopes et al. ${ }^{22}$, procurou investigar quais eram as expectativas de mulheres primíparas de Porto Alegre - RS antes e após o parto normal ou cesárea. Assim, foram entrevistadas 28 
mulheres e os resultados mostraram que a maioria delas possuía expectativas negativas antes do parto como: receio de que o bebê nasça prematuro, dor, insegurança e até mesmo o medo de morrer justificadas principalmente pela prestação de serviços assistenciais pouco acolhedores. Contudo, após o nascimento, a grande maioria relatou a experiência como excelente, justificada pela tranquilidade vivenciada no período anterior ao parto e pela qualidade e rapidez do atendimento. Os autores concluíram que não houve diferenças entre os tipos de parto em relação às expectativas antes e após o parto, entretanto, é necessária uma maior sensibilização e posição acolhedora na relação profissional de saúde - gestante, tendo em vista a fragilidade que as parturientes apresentam neste momento de suas vidas ${ }^{22}$.

Segundo Souza ${ }^{23}$, mesmo cidades como Belo Horizonte, que apresenta grandes avanços em relação ao processo de humanização do parto e sua assistência, o Brasil não atingirá a meta do milênio, que seria do índice "Razão de Mortalidade Materna” (RMM), igual ou inferior a 35 mortes por 100.000 nascidos vivos em 2015. Em Belo Horizonte a maior parte dos óbitos ocorreu no período pós-parto. Entretanto, Resende et al. ${ }^{24}$ afirmam que a atenção qualificada, captação precoce da gestante no pré-natal e a valorização das queixas das mulheres são fatores fundamentais para evitar mortes maternas.

De acordo com a Prefeitura Municipal de Belo Horizonte ${ }^{25}$, o debate sobre a influência da cesariana sem indicação clínica, que pode inclusive resultar em morte materna, é acirrado. No Brasil, há uma clara tendência de aumento desse tipo de cirurgia, especialmente no sistema privado, fato que preocupa profissionais de saúde pública devido à intercorrências negativas associadas a esse procedimento. Essa também é uma realidade em Belo Horizonte, onde 77,8\% dos partos realizados em hospitais privados são cesarianas $^{25}$.

\section{Conclusão}

É sabido que o aumento nas taxas de parto cesáreo é um fenômeno mundial desde as últimas décadas do século $\mathrm{XX}^{1}$. Além disso, em muitos locais do mundo a realização dessa cirurgia são superiores aquelas preconizadas pela $\mathrm{OMS}^{2}$. Porém, o "bom parto", seja ele vaginal, seja cesáreo, deve ser aquele que assegure o bem-estar da mãe e do recém-nascido. As decisões pelo tipo de parto devem considerar as preferências das gestantes, desde que elas tenham condições de escolher, de forma isenta, aquele que melhor lhes convém.

De acordo com os trabalhos apresentados, ficou evidente que existe uma tentativa de oferecer uma assistência humanizada no período prévio ao parto, durante a realização do mesmo e no período puerperal, nas regiões sul e sudeste do Brasil. Entretanto, o processo de humanização ocorre de maneira heterogênea entre os estados que compõe as regiões analisadas. Contudo, podemos observar uma fragilidade dos sistemas de saúde, nas diferentes regiões estudadas, em oferecer um atendimento precoce para a população mais pobre, seja essa uma assistência humanizada ou não.

Considerando a existência de evidências científicas que comprovam cada vez mais os benefícios de uma assistência humanizada, é fundamental que a formação acadêmica incorpore as mudanças no paradigma de atenção à saúde da mulher de maneira integral. Assim, se faz necessário a incorporação de novas atitudes por parte dos profissionais, que vise uma assistência multidisciplinar, com enfoque ao trabalho em equipe, que garanta a saúde e os direitos das mulheres e de seus recém-nascidos; o preparo da gestante para o parto desde o pré-natal; além da efetiva incorporação do modelo humanizado de atenção como diretriz e filosofia institucional buscando respeitar a fisiologia da mulher e favorecer os interesses de todos os envolvidos. 


\section{Colaboradores}

RM Pereira trabalhou no levantamento bibliográfico, na concepção e na redação final do artigo; GO Fonseca trabalhou no levantamento bibliográfico, concepção do abstract e revisão crítica do artigo; ACCC Pereira trabalhou no levantamento bibliográfico e formatação do artigo; GA Gonçalves trabalhou no levantamento bibliográfico e adequação do artigo as normas da revista C\&SC; RA Mafra trabalhou na correção ortográfica do artigo e aprovação da versão final a ser publicada.

\section{Referências}

1. Patah LEM, Malik AM. Modelos de assistência ao parto e taxa de cesárea em diferentes países. Rev Saude Publica 2011; 45(1):186-194.

2. Barros FC, Vaugham JP, Victora CG. Why so many caesarean sections? The need for a further policy change in Brazil. Health Policy Plan 1986; 1(1):19-29.

3. Barbosa GP, Giffin K, Angulo-Tuesta A, Gama AS, Chor D, D’Orsi E, Reis ACGV. Parto cesáreo: quem o deseja? Em quais circunstâncias? Cad Saude Publica 2003; 19(6):1611-1620.

4. Bergholt T, Ostberg B, Legarth J, Weber T. Danish obstetricians personal preference and general attitude to elective cesarean section on maternal request: A nation-wide postal survey. Acta Obstet Gynecol Scand 2004; 83(3):262-266.

5. Lurie S. The changing motives of cesarean section: from the ancient world to the twenty-first century. Arch Gynecol Obstet 2005; 271(4):281-285.

6. ANS Online: Novos modelos de atenção ao parto. Qualidade da Saúde. [acessado 2015 Nov 10]. Disponível em: http://www.ans.gov.br/aans/noticias-ans/ qualidade-da-saude/2734-novos-modelos-de-atencao -ao-parto

7. Rattner D. Humanização na atenção a nascimentos e partos: breve referencial teórico. Interface (Botucatu) 2009; 13(1):595-602.

8. Dias MAB, Domingues R. Desafios na implantação de uma política de humanização da assistência hospitalar ao parto. Cien Saude Colet 2005; 10(3):699-705.

9. Borges MR, Madeira LM, Azevedo VMGO. As práticas integrativas e complementares na atenção à saúde da mulher: uma estratégia de humanização da assistência no Hospital Sofia Feldman. Rev Min Enferm 2011; 15(1):105-113.

10. Machado NXS, Praça NS. Centro de parto normal e assistência obstétrica centrada nas necessidades da parturiente. Rev Esc Enferm USP 2006; 40(2):274-279.

11. Schmidt N, Abelsen B, Qian P. Deliveries in maternity homes in Norway: results from a 2 year prospective study. Acta Obstet Gynecol Scand 2002; 81(8):731-737.

12. Jackson DJ, Lang JM, Swartz WH, Ganiats TG, Fullerton J, Ecker J, Nguyen U. Outcomes, safety, and resource utilization in a collaborative care birth center program Compared with traditional physician-based perinatal care. Am J Public Health 2003; 93(6):999-1006.

13. Campos SEV, Lana FCF. Resultados da assistência ao parto no Centro de Parto Normal Dr. David Capistrano da Costa Filho em Belo Horizonte, Minas Gerais, Brasil. Cad Saude Publica 2007; 23(6):1349-1359.

14. Hodnett ED. Pain and women's satisfaction with the experience of childbirth: a systematic review. Am J Obstet Gynecol 2002; 186(5):160-172.

15. Puccini RF, Pedroso GC, Silva EMK, Araújo NS, Silva NN. Equidade na atenção pré-natal e ao parto em área da Região Metropolitana de São Paulo, 1996. Cad Saude Publica 2003; 19(1):35-45.

16. Neumann NA, Tanaka OU, Victora CG, Cesar JA. Qualidade e equidade da atenção ao pré-natal e ao Parto em Criciúma, Santa Catarina, Sul do Brasil. Rev Bras Epidemiol 2003; 6(4):307-318.

17. Primo CC, Amorim MHC, Castro DS. Perfil Social e Obstétrico das Puérperas de uma Maternidade. Rev Enferm UERJ 2007; 15(2):161-167. 
18. Leite FMC, Barbosa TKO, Bravim LR, Amorim MHC, Primo CC. A influência das características socioeconômicas no perfil obstétrico de puérperas. Aquichan 2014; 14(4):571-581.

19. D’Orsi E, Chor D, Giffin K, Angulo-Tuesta A, Barbosa GP, Gama AS, Reis AC, Hartz Z. Qualidade da atenção ao parto em maternidades do Rio de Janeiro. Rev Saude Publica 2005; 39(4):646-654.

20. Marque FC, Dias IMV, Azevedo L. A Percepção da Equipe de Enfermagem sobre a Humanização do Parto e Nascimento. Esc Anna Nery Rev Enferm 2006; 10(3):439-447.

21. Nagahama EEI, Santiago SM. Práticas de atenção ao parto e os desafios para humanização do cuidado em dois hospitais vinculados ao Sistema Único de Saúde em município da Região Sul do Brasil. Cad Saude Publica 2008; 24(8):1859-1868.

22. Lopes RCS, Donelli TS, Lima CM, Piccinini CA. O Antes e o Depois: Expectativas e Experiências de Mães sobre o Parto. Psicologia: Reflexão e Crítica 2005; 18(2):247-254.

23. Souza JP. Mortalidade materna e desenvolvimento: a transição obstétrica no Brasil. Rev Bras Ginecol Obstet 2013; 35(12):533-535.

24. Resende LV, Rodrigues RN, Fonseca MC. Mortes maternas em Belo Horizonte, Brasil: percepções sobre qualidade da assistência e evitabilidade. Rev Panam Salud Publica 2015; 37(4/5):218-224.

25. Belo Horizonte. Prefeitura Municipal. Boletim de Análise do Estado de Saúde da População Residente de Belo Horizonte: análise dos indicadores do nível municipal ciclo de vida - gestante. Belo Horizonte: Prefeitura Municipal de Belo Horizonte; 2011.

Artigo apresentado em 16/12/2015

Aprovado em 13/02/2017

Versão final apresentada em 15/02/2017 